\title{
Arbeitsmarktpolitik für ältere Arbeitnehmerlnnen im Wohlfahrtsstaatenvergleich
}

\author{
Frerich Frerichs
}

\begin{abstract}
Als Reaktion auf die demografischen Herausforderungen, die sich für den Arbeitsmarkt stellen, sind in jüngerer Zeit in zahlreichen westlichen Industriestaaten arbeitsmarktpolitische Handlungsansätze für ältere ArbeitnehmerInnen entwickelt worden. In den begleitenden Debatten wird bisher wenig Bezug auf die zugrunde liegenden, unterschiedlichen wohlfahrtsstaatlichen Strukturen genommen und stattdessen im Sinne eines „,one size fits all“-Ansatzes oft davon ausgegangen, dass sich die jeweiligen Programmatiken im Sinne eines „active ageing“ einander annähern sollten. Der folgende Beitrag stellt demgegenüber die Eigenlogiken der Wohlfahrtsstaatensysteme heraus und analysiert kritisch die daraus hervorgehende, je spezifische Form der Bekämpfung der Arbeitsmarktprobleme älterer ArbeitnehmerInnen.
\end{abstract}

\section{Einführung}

Die entwickelten Industriestaaten sehen sich aufgrund des generellen Trends steigender Lebenserwartung und sinkender Geburtenraten einer zukünftig älter werdenden und schrumpfenden (Erwerbs-) Bevölkerung gegenüber. In der Folge müssen die wirtschaftlich-technologischen Entwicklungen zunehmend von älteren ArbeitnehmerInnen gestaltet werden. Zudem gilt neben der Erhöhung der Produktivität und einer Ausweitung der Frauenerwerbsarbeit die verstärkte Integration älterer ArbeitnehmerInnen in die Erwerbsarbeit als Mittel der Wahl, um die prognostizierte Schrumpfung des Erwerbspersonenpotenzials abzufedern. Der Druck auf eine stärkere Integration älterer ArbeitnehmerInnen in das Erwerbsleben erhöht sich zusätzlich dadurch, dass aus rentenfinanzpolitischen Erwägungen heraus eine Anhebung der (Regel-)Altersgrenzen für den Renteneintritt in jüngster Zeit in mehreren Staaten - so auch in Großbritannien, Japan und Deutschland - erfolgt ist bzw. geplant wird.

Der doppelte Handlungsdruck auf die Arbeitsmarktpolitik steht in einem Spannungsverhältnis zu den Ausgliederungsprozessen und Beschäftigungsrisiken älterer ArbeitnehmerInnen am Arbeitsmarkt, die sich vor allem in einem überdurchschnittlichen Arbeitslosigkeitsrisiko und einer unterdurchschnittlichen Erwerbsbeteiligung ausdrücken. Im EU- und OECDRaum sind ältere ArbeitnehmerInnen immer wieder als mehr oder minder große Problemgruppe am Arbeitsmarkt charak- terisiert und als Verfügungsmasse in arbeitsmarktpolitischen Krisensituationen instrumentalisiert worden (ETUI 2002; Maltby et al. 2004).

Vor diesem Hintergrund haben sich vielfältige arbeitsmarktpolitische Handlungsansätze herausgebildet, die auf eine stärkere Integration älterer ArbeitnehmerInnen in das Erwerbsleben abzielen. In aktuellen Bestandsaufnahmen werden diese Ansätze oft als beispielhafte best-practiceModelle herausgestellt und es wird relativ unbesehen von einer Übertragbarkeit auf andere Staaten ausgegangen (Taylor 2002, 2006; Bertelsmann Stiftung/BDA 2003; OECD 2006a; Prager/Schleiter 2006). Systematische und analytisch-vergleichende Studien, die die bestehenden Unterschiede in der arbeitsmarktpolitischen Ausrichtung vor dem Hintergrund wohlfahrtsstaatlicher Handlungstypen zu erklären versuchen und sich die Frage stellen, ob sich aus dem demografischen Handlungsdruck tatsächlich eine Konvergenz der Politikmuster ergeben kann, sind dagegen eher selten.

Im Folgenden sollen daher exemplarisch die Entwicklungsmuster der aktiven Arbeitsmarktpolitik für ältere ArbeitnehmerInnen in Großbritannien, Japan und Deutschland auf Basis von kontrastiven Fallstudien verglichen und die unterschiedlichen wohlfahrtsstaatlichen Leistungsprofile analysiert werden. ${ }^{1}$ Dadurch soll die Spannbreite der möglichen arbeitsmarktpolitischen Antworten auf die demografischen Herausforderungen aufgezeigt und gleichzeitig verdeutlicht werden, mit welchen je spezifischen Chancen und Risiken für ältere ArbeitnehmerInnen diese einhergehen. Der Wohlfahrtsstaatenvergleich ist hierbei bipolar angelegt: Gegen- übergestellt werden mit Großbritannien und Deutschland ein marktliberaler und ein konservativ-korporatistischer Wohlfahrtsstaat, Japan wird als „hybrider" Mix aus liberalem und konservativ-korporatistischem Wohlfahrtsstaat gewertet und nimmt damit eine Mittelstellung zwischen den beiden anderen Staaten ein (EspingAndersen 1990, 1997, 1999).

\section{Demografischer Wandel und Arbeitsmarkt - Die Ausgangssituation}

Die demografischen Entwicklungen treffen in Großbritannien, Japan und Deutschland nicht auf einen ,altersneutralen“ Arbeitsmarkt, sondern es sind jeweils spezifische Segmentierungen und gravierende altersbezogene Arbeitsmarktrisiken zu konstatieren.

\footnotetext{
1 Der Vergleich Großbritannien - Deutschland baut auf den Ergebnissen eines von der Anglo-German Foundation geförderten Projektes mit dem Titel „Labour market policies for older workers and demografic change" auf (Frerichs/Taylor 2005).
}

Frerich Frerichs, Prof. Dr., Institut für
Gerontologie der Hochschule Vechta,
Lehrstuhl "Altern und Arbeit“. Arbeits-
schwerpunkte: Betriebliche Beschäftigungs-
politik und alternde Belegschaften,
Arbeitsmarktpolitik für ältere Arbeit-
nehmerlnnen.
e-mail: frerich.frerichs@uni-vechta.de




\subsection{GROßBRITANNIEN: ALTERUNG UND ARBEITSMARKTBEDINGTE INAKTIVTÄT}

In Großbritannien trifft das Altern der Erwerbsbevölkerung - den vorliegenden Prognosen zufolge steigt der Anteil der 50- bis 64-Jährigen an der Erwerbsbevölkerung von $27 \%$ im Jahr 2000 auf $32 \%$ im Jahr 2020 (Barham 2002) - auf Beschäftigungsverhältnisse von älteren ArbeitnehmerInnen, die durch einen zunehmenden Wechsel von einer unbefristeten Vollzeiterwerbsarbeit in Teilzeitbeschäftigung, selbstständige Tätigkeiten und befristete Beschäftigungsformen gekennzeichnet sind (Hirsch 2003). Dies kann als Zeichen für die auf dem britischen Arbeitsmarkt wirkenden Beschäftigungshürden für ältere ArbeitnehmerInnen gewertet werden, die nicht zuletzt aus ihrem durchschnittlich sehr viel niedrigeren Qualifikationsniveau resultieren. In Großbritannien sind zudem infolge wirtschaftlicher Strukturkrisen die Erwerbsquoten älterer ArbeitnehmerInnen in den 1970er, 1980er und 1990er Jahren stark zurückgegangen, und auch die Altersarbeitslosigkeit war ausgeprägt (Walker 2002). Aufgrund des seit Ende der 1990er Jahre anhaltenden Wirtschaftsaufschwungs ist diese zwar wieder auf einen niedrigen Stand gesunken und auch die Erwerbsquoten der 55- bis 59-jährigen Männer haben mit ca. $75 \%$ und die der gleichaltrigen Frauen mit ca. 60 \% wieder ein höheres Niveau erreicht (OECD 2004a); dennoch liegt weiterhin ein erhebliches Maß an arbeitsmarktbedingter Inaktivität bei älteren ArbeitnehmerInnen vor, die sich insbesondere im Bezug von Erwerbsunfähigkeitsrenten sowie in einer verfestigten Langzeitarbeitslosigkeit ausdrückt (Frerichs/Taylor 2005). Zudem spiegeln die Alterserwerbsquoten nicht den hohen Grad an Teilzeitbeschäftigung wider; bei Umrechnung auf Vollzeiterwerbsquoten liegt die Erwerbsbeteiligung bedeutend niedriger (OECD 2004b).

\subsection{JAPAN: ALTERUNG, SCHRUMPFUNG UND BETRIEBLICHE ALTERSGRENZEN}

Japan ist durch einen rapiden Alterungsprozess in der Arbeitswelt gekennzeichnet: Im Jahr 2020 werden bereits rd. $45 \%$ der Erwerbsbevölkerung zwischen 45 und 64 Jahre alt sein (AEDSC 2002). Zudem zeichnet sich Japan gegenüber Deutschland und
Großbritannien durch einen sehr früh, nämlich bereits ab 2007 einsetzenden Schrumpfungsprozess in der (Erwerbs-) Bevölkerung aus (MHLW 2005). Dieser demografischen Entwicklung steht in Japan ein stark segmentierter Arbeitsmarkt gegenüber. Zum einen besteht ein Kernsegment der „lebenslangen Beschäftigung“, in dem vorwiegend männliche Beschäftigte in Großbetrieben tätig sind und das sich durch gute Aufstiegs- und Qualifizierungsmöglichkeiten, der Partizipation an Sozialleistungen des Betriebs und vor allem durch eine weitgehende Beschäftigungsgarantie bis zum Erreichen der betrieblichen Altersgrenzen auszeichnet (Araki 2002; Casey 2006). Den vorliegenden Schätzungen zufolge liegt der Anteil zwischen $20 \%$ und $30 \%$ aller Beschäftigten (Pascha 2003). Doch bereits in diesem Kernsegment herrscht eine altersdiskriminierende Praxis vor: Nach Erreichen der stark institutionalisierten betrieblichen Altersgrenzen - über $90 \%$ der Betriebe praktizieren dieses System, die Altersgrenzen sind in der Regel auf 60 Jahre festgelegt (MHLW 2005) - erfolgt der Transfer in Unternehmensbereiche mit weitgehend ungesicherten und deutlich geringer vergüteten Beschäftigungsverhältnissen (Seike 2001; Casey 2006). Diesem Kernsegment des Arbeitsmarktes steht ein ohnehin stark von ungesicherten Beschäftigungsformen geprägter Sektor gegenüber, mit dem ältere Erwerbstätige in besonderem Maße konfrontiert sind. Sie werden mit zunehmendem Alter deutlich häufiger auf eine selbstständige Tätigkeit verwiesen und befinden sich weitaus öfter in Teilzeitbeschäftigung: Nahezu ein Viertel der 60bis 64-jährigen Arbeitnehmer und fast die Hälfte der gleichaltrigen Arbeitnehmerinnen sind teilzeitbeschäftigt (OECD 2001). Dieser Status bringt in Japan eine deutlich schlechtere arbeitsrechtliche Stellung und einen überproportional niedrigen Lohn mit sich (AEDSC 2002; Shire 2003).

In Japan sind zwar in der Vergangenheit die Erwerbsquoten der älteren ArbeitnehmerInnen auf hohem Niveau relativ stabil geblieben, allerdings zeichnet sich auch hier in der Gruppe der 60- bis 64-Jährigen ein sukzessiver Rückgang ab (AEDSC 2002). Im Zuge der seit Beginn der 1990er Jahre andauernden Rezessionsphase ist zudem die Altersarbeitslosigkeit auf für japanische Verhältnisse ungewöhnlich hohe Werte von rd. $5 \%$ für 55 - bis 59 -jährige und von $10 \%$ für 60- bis 64-jährige Männer angestiegen (AEDSC 2002). Nicht ent- halten darin ist das hohe Maß an Unterbeschäftigung älterer ArbeitnehmerInnen in den Betrieben (Waldenberger 2003).

\subsection{DEUTSCHLAND: ALTERUNG UND NIEDRIGE ERWERBSBETEILIGUNG}

Der Alterungsprozess der Erwerbsbevölkerung ist in Deutschland ebenfalls sehr ausgeprägt - der Anteil der 50- bis 64-Jährigen an der Erwerbsbevölkerung steigt von $30 \%$ im Jahr 2000 auf $39 \%$ im Jahr 2020 -, allerdings ist erst in mittelfristiger Perspektive - ab dem Jahr 2020 - mit einem Rückgang der Erwerbsbevölkerung zu rechnen (Statistisches Bundesamt 2003). Auf dem Arbeitsmarkt zeigt sich eine deutliche geschlechts- und qualifikationsspezifische Segmentierung für ältere ArbeitnehmerInnen. So sind nahezu $55 \%$ der 55-jährigen und älteren weiblichen, aber nur rd. $10 \%$ der gleichaltrigen männlichen Erwerbstätigen in Westdeutschland teilzeitbeschäftigt (Hoffmann/Walwei 2002). Diese Beschäftigungsform geht zwar nicht mit einem generell schlechteren arbeitsrechtlichen Status wie in Japan einher, bewirkt aber dennoch deutlich geringere Aufstiegs- und Verdienstchancen. Die Integration in Erwerbsarbeit ist zudem stark nach Qualifikationsstufen segmentiert, und die daraus resultierende Benachteiligung niedrig Qualifizierter nimmt mit dem Alter deutlich zu. Während z. B. in der Gruppe der 50- bis 64-Jährigen die Diskrepanz in der Erwerbsbeteiligung zwischen der niedrigsten und der höchsten Qualifikationsstufe mehr als 30 Prozentpunkte beträgt, liegt diese bei den ArbeitnehmerInnen in der Altersgruppe von 25 bis 49 Jahren nur bei knapp 20 Prozentpunkten (OECD 2003). Infolge wirtschaftlicher Strukturkrisen und zusätzlich durch den Sondereffekt der Wiedervereinigung sind die Alterserwerbsquoten bis Mitte/Ende der 1990er Jahre vor allem bei den Männern stark gesunken. Erst in jüngerer Zeit zeichnet sich wieder ein leichter Anstieg ab. Dieser relativiert sich allerdings sehr stark vor dem Hintergrund der hohen Arbeitslosenquoten, die in den Altersgruppen von 50 bis 54 Jahren und von 55 bis 59 Jahren bei rd. 16 \% (Männer) bzw. rd. $17 \%$ (Frauen) liegen (BA 2003).

Die skizzierten Arbeitsmarktrisiken und -segmentierungen erschweren eine verstärkte Nutzung des Arbeitskraftpotenzials älterer ArbeitnehmerInnen, die vor dem Hintergrund der Alterung der Erwerbsbevölkerung und der Anhebung der 
Altersgrenzen erforderlich wäre. Die zum Teil erst später einsetzende Schrumpfung des Erwerbspersonenpotenzials bringt zudem noch keine angebotsbedingte Entlastung des Arbeitsmarktes mit sich, sodass auch nicht von einem demografisch bedingten Automatismus beim Abbau von Arbeitsmarktrisiken ausgegangen werden kann.

\section{Aktive Arbeitsmarktpolitik für ältere Arbeitnehmer- Innen im Wohlfahrtsstaat}

Die aufgezeigten Entwicklungstendenzen bezüglich der Alterung und Schrumpfung der Erwerbsbevölkerung und die Arbeitsmarktprobleme älterer ArbeitnehmerInnen, die diesen gegenüberstehen, sind auf Ebene der staatlichen Arbeitsmarktpolitik in allen drei Ländern erst seit jüngerer Zeit Gegenstand von Reformüberlegungen. Versuche, über die Anhebung der Altersgrenzen Einfluss auf die Steigerung der Erwerbsquoten und das faktische Rentenzugangsalter zu nehmen, reichen zwar bis Anfang der 1990er Jahre zurück, sie sind aber wesentlich auf rentenfinanzpolitische Überlegungen zurückzuführen. Zusehends wird jetzt deutlich, dass ergänzend hierzu arbeitsmarktpolitische Maßnahmen ergriffen werden müssen, wenn den demografischen Herausforderungen wirkungsvoll begegnet werden soll.

Im Vereinigten Königreich ist von der Labour-Regierung erstmals im Jahr 2000 mit dem Strategie-Papier „Winning the Generation Game“ explizit auf die demografischen Herausforderungen im Bereich der Arbeitsmarktpolitik Bezug genommen worden (Cabinet Office 2000). Ein Teil der dort getroffenen insgesamt 75 (!) arbeitsmarktpolitischen Handlungsempfehlungen wird in dem im gleichen Jahr eingeführten New Deal 50plus aufgegriffen. In mehreren aufeinander folgenden programmatischen Papieren - insbesondere in dem im Jahr 2002 vorgelegten Grünbuch zur Altersvorsorge (DWP 2002a ) und zuletzt im Jahr 2006 mit dem „New Deal for Welfare Empowering People to work" (DWP 2006) - werden die daraus hervorgegangenen Maßnahmen weiterentwickelt.

In Deutschland fand im Jahr $2001 \mathrm{mit}$ der gemeinsamen Erklärung des Bündnisses für Arbeit und Wettbewerbsfähigkeit ein Paradigmenwechsel statt, der auf einen Ausbau aktiver arbeitsmarktpolitischer Instrumente und einen Stop des bisherigen Frühverrentungstrends abzielte (Gemeinsame Erklärung 2001). Ihren ersten sichtbaren Ausdruck fand dieser Richtungswechsel im Job-AQCTIV-Gesetz, das im darauffolgenden Jahr verabschiedet wurde. Im Zuge der Hartz-Reformen wurden mehrere neue Instrumente zur Beschäftigungsförderung älterer ArbeitnehmerInnen aufgelegt - allerdings eingebunden in eine generelle Neuausrichtung der Arbeitsmarktpolitik, die Problemgruppen eher benachteiligte (Abschnitt 3.2). Den bisherigen Schlusspunkt bildet die 2006 verkündete „Initiative 50plus“ des Arbeitsministeriums, die bestehende arbeitsmarktpolitische Ansätze für ältere ArbeitnehmerInnen zu bündeln und zu erweitern sucht (BMAS 2006).

In Japan wird mit dem im Jahr 2000 vorgelegten Weißbuch zur Arbeitsmarktpolitik verstärkt auf die demografischen Herausforderungen aufmerksam gemacht und ein längerer Verbleib im Erwerbsleben gefordert (Ministry of Labour 2000). Bereits im Jahr zuvor waren mit dem „9. Maßnahmenprogramm zur Stabilisierung der Beschäftigung und zur Schaffung neuer Beschäftigungsmöglichkeiten" Befürchtungen hinsichtlich möglicher negativer Auswirkungen der Bevölkerungsalterung auf die wirtschaftliche Prosperität geäußert worden, und es wurde die Zielstellung vorgegeben, eine „altersfreie“, das heißt nicht altersdiskriminierende Arbeitsmarkt- und Beschäftigungspolitik zu erreichen (Iwata 2002). Im Weißbuch zur Arbeitsmarktpolitik aus dem Jahr 2005 wird schließlich auf das Hineinwachsen der japanischen Baby-Boom-Generation in rentennahe Jahrgänge verwiesen und auf die daraus hervorgehenden Anforderungen an eine altersgerechte Gestaltung der Arbeitswelt aufmerksam gemacht (MHLW 2005).

Bereits an dieser Stelle muss darauf hingewiesen werden, dass die arbeitsmarktpolitischen Programmatiken in ihrer Stoßrichtung deutlich voneinander abweichen. Während die Labour Regierung in Großbritannien im Wesentlichen auf die Aktivierung arbeitsmarktferner Gruppen in der älteren Erwerbsbevölkerung abzielt, sieht sich Deutschland vor die Herausforderung gestellt, in Anbetracht der bereits vollzogenen Altersgrenzenanhebung diese Entwicklung auch arbeitsmarktpolitisch „einzuholen“ und nimmt ältere Arbeitneh-
merInnen generell in den Fokus. Japan hingegen steht angesichts des gravierenden Alterungsprozesses in der Erwerbsbevölkerung der Situation gegenüber, auf die zunehmende Betroffenheit älterer ArbeitnehmerInnen von betrieblichen Altersgrenzen zu reagieren und zugleich die hohe Altersarbeitslosigkeit zu bekämpfen.

\subsection{ARBEITSMARKTPOLITISCHE MABNAHMEN IM VERGLEICH}

Der aktiven Arbeitsmarktpolitik kommt eine zentrale Rolle zu, die durch den demografischen Wandel geforderte stärkere Nutzung der Humanressourcen älterer ArbeitnehmerInnen sicherzustellen. Die folgenden vergleichenden Analysen zielen darauf, die wesentlichen Unterschiede und Gemeinsamkeiten der arbeitsmartkpolitischen Maßnahmen für ältere ArbeitnehmerInnen in Großbritannien, Japan und Deutschland herauszustellen und in wohlfahrtsstaatliche Handlungsmuster einzuordnen.

\section{ARBEITSVERMITTLUNC}

Das Arbeitslosigkeitsregime in Großbritannien zeichnet sich durch eine starke Konzentration auf sanktionsbewährte Vermittlungsaktivitäten aus. In den ersten sechs Monaten der Arbeitslosigkeit bleiben ältere Arbeitslose dabei nach wie vor von aktiven arbeitsmarktpolitischen Maßnahmen ausgeschlossen und sind auf die allgemeine Vermittlung im Rahmen der JobcentrePlus verwiesen sowie in zeitlich eng hintereinander geschaltete Beratungsgespräche und verpflichtende Aktivitäten zur Arbeitssuche eingebunden. Vermittlungsanstrengungen für ältere Arbeitslose werden im Rahmen von, ,job entry targets “ formal zwar höher bewertet, das strikte Vermittlungsregime bringt allerdings auch das Risiko der Vermittlung in niedrig qualifizierte, schlechter entlohnte Jobs mit geringen Aufstiegsmöglichkeiten mit sich (Atkinson et al. 2003). Zudem gelten die Anzahl und insbesondere die Qualifizierung des Beratungspersonals in der Arbeitsvermittlung als verbesserungsbedürftig, und es liegt eine relativ hohe, die Kontinuität der Beratung infrage stellende Personalfluktuation vor (Comptroller and Auditor General 2004). Nach sechsmonatiger Arbeitslosigkeit können ältere Arbeitslose dagegen im Rahmen des New Deal 50plus und bei länger als 18 Monate anhaltender 
Arbeitslosigkeit auch im Rahmen des New Deal 25plus besondere und zum Teil wöchentlich verfügbare Beratungsangebote und Assessments in Anspruch nehmen, die zudem als Schlüssel zu weiteren Förderaktivitäten wie Qualifizierungsprogrammen und Einkommenszuschüssen fungieren. Vor dem Hintergund des hohen Bestandes an Erwerbsunfähigen versucht die britische Regierung seit jüngerer Zeit des Weiteren, Anreize und Fördermöglichkeiten für eine Wiedereingliederung in das Erwerbsleben für diese Gruppe zu entwickeln (DWP 2003, 2006). Diese arbeitsmarktpolitischen Interventionen erfolgen aber eher zögerlich und auf einem niedrigen finanziellen und materiellen Niveau (Frerichs/Taylor 2005).

Auch in Deutschland weist die Arbeitsvermittlung und -beratung der lokalen Arbeitsagenturen bezogen auf die - gesetzlich verankerte - verstärkte Unterstützung von Problemgruppen noch erhebliche Defizite auf. Zwar sind mit der Durchführung von Profiling-Maßnahmen prinzipiell gute Möglichkeiten gegeben, den Vermittlungsprozess besser auf Bedürfnisse und Möglichkeiten älterer Arbeitsloser abzustellen. Die gegenwärtig noch unzureichenden Personalressourcen wirken dem aber genauso entgegen wie die geschäftspolitische Zielsetzung der Bundesagentur für Arbeit (BA) in Richtung auf einen schnellen Eingliederungserfolg (OECD 2005, 2006b; Deutscher Bundestag 2006). Diese Geschäftspolitik führt zu sogenannten „Creaming-Effekten", das heißt, in der Regel wird die Vermittlung auf ein jüngeres bzw. leichter zu vermittelndes Klientel ausgerichtet (Schütz/Oschmiansky 2006). Die ebenfalls als vermittlungsaufschließendes Instrument - aber auch als Kontrollelement zur Überprüfung der Arbeitsbereitschaft - eingeführten Trainingsmaßnahmen zeigen ebenfalls einen deutlichen Altersbias (BA 2004).

Angesichts der in der letzten Dekade drastisch gestiegenen Arbeitslosigkeit unter den älteren ArbeitnehmerInnen kommt auch in Japan der Arbeitsvermittlung für diese Zielgruppe eine hohe Bedeutung zu. Besondere Vermittlungsaktivitäten für ältere Arbeitslose sind allerdings primär auf höher qualifizierte und infolge des Erreichens der betrieblichen Altersgrenzen bzw. der Versetzung in ungeschützte Beschäftigungsverhältnisse arbeitslos gewordene ältere Fachkräfte ausgerichtet und umfassen zumeist nur Be- werbungs- und Berufslaufbahnberatung (Naganawa 2002; OECD 2004c). Es fehlen insbesondere Vermittlungsangebote für ältere Langzeitarbeitslose oder niedrig qualifizierte ältere Arbeitslose, etwa in Form von Profiling-, Trainings- oder mobilitätsfördernden Maßnahmen. Insgesamt muss die Vermittlungsarbeit für ältere Arbeitslose daher als stark segmentierend gewertet werden.

\section{BERUFLICHE QUALIFIZIERUNG}

Qualifizierungsmaßnahmen und die Förderung lebenslangen Lernens nehmen sowohl in Großbritannien als auch in Deutschland auf der programmatischen Ebene einen hohen Stellenwert ein. In Großbritannien ist allerdings in der Realität nur eine gering entwickelte Angebotsstruktur bezogen auf die Qualifizierungsförderung von (älteren) Arbeitslosen auszumachen. Die im Rahmen des New Deal 50plus gezielt für ältere Arbeitslose gewährten "training grants" sind finanziell mit Fördersummen von rd. $2.400 €$ nur relativ gering ausgestattet. Und sie werden auch nur für Qualifizierungen gewährt, die nach Arbeitsaufnahme erfolgen. In der Konsequenz nehmen nur rd. $5 \%$ aller TeilnehmerInnen des Programms New-Deal 50plus entsprechende Fördermöglichkeiten in Anspruch (DWP 2002b). Im Rahmen des Work based Learning for Adults (WBLA) wird des Weiteren eine Mischung aus kurzen Trainings- und längeren Weiterbildungsmaßnahmen für alle Arbeitslosen angeboten. Mit jährlichen Teilnehmereintritten von 60.000 Personen bleibt diese Fördermaßnahme aber deutlich hinter den bundesrepublikanischen Zahlen zurück: Im Jahr 2003 - also bereits nach Einsetzen der Förderreduzierung im Zuge der HartzReformen - betrugen die jährlichen Eintritte in Trainings- und Weiterbildungsmaßnamen zusammengenommen immer noch rd. 1,2 Mio. Personen (Frerichs/Taylor 2005). Auch die niedrigere Arbeitslosenquote im Vereinigten Königreich vermag diesen Unterschied nicht zu erklären. Die Teilnehmerquoten von älteren (Langzeit-)Arbeitslosen liegen beim WBLA mit rd. $17 \%$ zwar relativ hoch, den vorliegenden Befunden zufolge partizipieren sie jedoch weniger an den umfangreicheren Weiterbildungsmaßnahmen in diesem Programm, die eine bessere Vermittlung in qualifizierte Tätigkeiten gewährleisten (DfEE 2001).
Die Problematik in der Förderung der beruflichen Weiterbildung älterer ArbeitnehmerInnen in der Bundesrepublik liegt nicht primär in fehlenden Angeboten, sondern in deren Ausgrenzung aus den bestehenden Maßnahmen: Nur rd. $5 \%$ aller TeilnehmerInnen an diesen Maßnahmen waren im Jahr 200350 Jahre und älter, obwohl deren Anteil an den Arbeitslosen im selben Zeitraum bei $25 \%$ lag (BA 2004). Zum einen wirkt hier die langjährige Frühverrentungspolitik weiter fort, die in den Augen der Arbeitsverwaltung Qualifizierungsmaßnahmen für ältere Arbeitslose wenig lohnend erscheinen lässt. Zum anderen ist hierfür die im Zuge der Hartz-Reformen eingeführte neue Geschäftspolitik der Bundesagentur für Arbeit verantwortlich zu machen, die sich an hohen und von älteren Arbeitslosen in der Regel nicht zu erreichenden Eingliederungsquoten von 70 \% für die Gewährung von Fördermitteln orientiert (Expertenkommission 2004; OECD 2005). Allerdings ist auch für die Bundesrepublik zu konstatieren, dass das ehemals sehr hohe Niveau an Weiterbildungsförderung stark reduziert worden ist und sich zusehends auf kurzfristige Maßnahmen konzentriert. Gesondert eingeführte Förderungen für die betriebliche Weiterbildung älterer ArbeitnehmerInnen in Klein- und Mittelbetrieben, die einen eher präventiven Charakter tragen, konnten bisher noch keine Wirksamkeit entfalten (Sproß/Eichhorst 2005).

In Japan ist aufgrund der auf die betriebliche Beschäftigungssicherung ausgerichteten Arbeitsmarktpolitik die Weiterbildungsförderung für ältere Arbeitslose weitgehend defizitär. Dies steht im Gegensatz zu der Tatsache, dass für die in der letzten Dekade stark angestiegene Zahl der älteren Arbeitslosen die mangelnde Transferierbarkeit von betrieblich erworbenen Qualifikationen im Erwerbsverlauf ein schwerwiegendes Beschäftigungs- und Eingliederungshemmnis darstellt (Naganawa 2002). Lediglich im Rahmen der allgemeinen Weiterbildungsförderung und auf einem sehr niedrigen finanziellen Niveau stehen älteren Arbeitslosen Qualifizierungsangebote zur Verfügung. Hierbei mangelt es zudem an einer Steuerung zwischen den angebotenen Maßnahmen und dem tatsächlichen Arbeitsmarktbedarf (Higuchi 2004). Die betriebsbezogene Weiterbildung wird in Japan seit 1998 im Rahmen des „Education and training benefit" individuell mit relativ geringen Beträgen geför- 
dert. 55-jährige und ältere ArbeitnehmerInnen sind aber - nicht zuletzt wegen der vorherrschenden betrieblichen Altersgrenzen - mit einer Teilnahmequote von $9 \%$ nur stark unterproportional in diesen Maßnahmen vertreten (OECD 2004c).

\section{LOHNSUBVENTIONIERUNG UND EINKOMMENSZUSCHÜSSE}

In Deutschland stellen Lohnsubventionen in Form von Eingliederungszuschüssen ein traditionell stark ausgebautes Instrument der aktiven Arbeitsmarktpolitik dar. Hierbei gelten zudem besondere Förderbestimmungen für ältere Arbeitslose ab 50 Jahren mit Förderdauern von derzeit maximal drei Jahren und degressiv gestalteten Fördersummen in Höhe von maximal 50 \% des berücksichtigungsfähigen Arbeitsentgeltes. Im Zeitraum der letzten Jahre ist dieses Förderinstrument zudem weiter ausgebaut worden und es werden derzeit rd. 150.000 Förderfälle pro Jahr registriert (BA 2004). Eine derartige Förderstruktur und -intensität der Lohnsubventionierung ist in Großbritannien nicht vorzufinden. Im Rahmen des New Deal 50plus existieren keine, im Rahmen des New Deal 25plus nur sehr begrenzte Möglichkeiten zur Gewährung von Lohnsubventionen für ältere Arbeitslose. Lohnsubventionen für ältere Arbeitslose werden auch in Japan, z. B. im Rahmen des „Subsidy for Employment Development for Special Type of Job-Seeker", gezahlt (Iwata 2002). Die Förderzahlen gehen aber ebenfalls nicht über einige Tausend Personen hinaus, das genannte Förderprogramm ist zudem finanziell sehr beschränkt (Othake 2004).

Diese Förderleistungen bleiben weit hinter den Unterstützungszahlungen zurück, die die japanische Regierung ArbeitnehmerInnen gewährt, die noch in den Betrieben beschäftigt sind, die aber im Zuge des Erreichens betrieblicher Altersgrenzen Lohneinbußen hinnehmen müssen. Aktuell werden mit diesem „Employment Continuation Benefit for the Aged" jährlich rd. 140.000 ältere ArbeitnehmerInnen mit einer Fördersumme von insgesamt rd. 1 Mrd. $€$ subventioniert, die Bezugsdauer liegt mit zwei Jahren relativ hoch (Iwata 2002; OECD 2004c). Die Maßnahme stellt im Prinzip eine staatliche Subventionierung der betrieblichen Ausgliederungspraxis („teinen“) dar. Lohneinbußen bei den älteren ArbeitnehmerInnen werden - wenigstens zum Teil - kompensiert, wenn die Wieder- bzw. Weiterbeschäftigung in demselben Betrieb, aber in sehr viel geringer entlohnten Tätigkeiten erfolgt.

Auch in Großbritannien sind als zentrales Instrument des New Deal 50plus Einkommenszuschüsse in Form des Employment Credits eingeführt worden, diese werden aber im Gegensatz zu Japan nur bei Einstellung von älteren Arbeitslosen gewährt. Mit diesen steuerfreien Zuschüssen zum Erwerbseinkommen in Höhe von rd. $385 €$ pro Monat und für die Dauer von einem Jahr sind im Zeitraum der ersten drei Jahre rd. 120.000 ältere Arbeitslose gefördert worden. Strukturell wird mit den Fördergegebenheiten die Aufnahme einer niedrig entlohnten Tätigkeit präjudiziert, und in der Folge lagen mehr als zwei Drittel der vermittelten Arbeitsplätze auf dem untersten Qualifikationsniveau mit sehr niedrigen Einkommen (Moss/Arrowsmith 2003). In Deutschland sind bezeichnenderweise Einkommenszuschüsse für ältere ArbeitnehmerInnen in Form der Entgeltsicherung erst relativ spät eingeführt worden, da es starken Widerstand gegen die Etablierung eines Niedriglohnsektors gab und gibt. In der jetzigen Form ${ }^{2}$ wird für die Dauer des Restanspruches auf Arbeitslosengeld ein Zuschuss zum Arbeitsentgelt in Höhe von 50 \% der monatlichen Differenz zum vorherigen Nettoentgelt gezahlt. Die damit angestrebte Absenkung des Reservationslohnes ist aber angesichts sehr geringer Förderzahlen bisher ohne großen Erfolg geblieben (Brussig et al. 2006).

\section{SCHAFFUNG ÖFFENTLICH GEFÖRDERTER ARBEITSGELEGENHEITEN}

Trotz des stärkeren Interventionsgrades der Labour-Regierung gegenüber der konservativen Vorgängerregierung sind Maßnahmen zur öffentlich geförderten Arbeitsplatzbeschaffung in Großbritannien bisher die Ausnahme geblieben und nicht auf ältere Arbeitslose zugeschnitten. Im Rahmen der New Deal-Programme werden diese lediglich für arbeitslose Jugendliche angeboten und haben eher einen die Arbeitsbereitschaft kontrollierenden Charakter. Insgesamt besteht nach wie vor aufgrund genereller arbeitsmarktpolitischer Erwägungen, die aus Bedenken gegen die staatliche Subventionierung von Arbeitsplätzen resultieren, eine große Reserviertheit gegenüber diesem Maßnahmetyp (Frerichs/Taylor 2005).
Nachfrageorientierte Strategien zur Arbeitsplatzbeschaffung sind dagegen in Deutschland nicht nur generell wesentlich stärker ausgeprägt, sie weisen auch seit jeher eine spezifische Ausrichtung auf ältere Erwerbslose als besondere Problemgruppe am Arbeitsmarkt aus. Allerdings ist auch hier infolge der Neuausrichtung der Geschäftspolitik der Bundesanstalt für Arbeit auf Maßnahmen mit schnellen Vermittlungserfolgen und im Rahmen eines , aktivierenden" Vermittlungsregimes die Förderung drastisch reduziert worden. Die Bestandszahlen sind von mehr als 300.000 auf unter 140.000 gesunken, wobei ältere Arbeitslose nicht zuletzt aufgrund der längeren Förderdauer anteilsmäßig zusehends stärker in diesen Maßnahmen vertreten sind (BA 2004). Im Rahmen der Überführung von Arbeitslosen in das Förderregime des SGB II nach einjähriger Arbeitslosigkeit sind ersatzweise befristete Arbeitsgelegenheiten, sogenannte Ein-EuroJobs, geschaffen worden, die im Vergleich zu ABM-Maßnahmen eine deutlich geringere Laufzeit und nur eine sehr geringe Aufstockung des bedarfsgeprüften Arbeitslosengelds II mit sich bringen. Hier schlägt sehr deutlich eine Re-Kommodifizierungstendenz in der arbeitsmarktpolitischen Förderung durch.

In Japan werden immer wieder ad-hoc Notfallmaßnahmen zur Arbeitsplatzbeschaffung, wie z. B. der 2001 eingeführte „Special Emergency Grant for Employment Creation" und das bereits im Jahr 1999 geschaffene Förderprogramm zur Schaffung von Arbeitsplätzen in Wachstumsbranchen („Special Grants for Creating Employment in New and Growth Sectors"), aufgelegt. Für ältere Arbeitslose werden in diesen Programmen aber in der Regel nur einmalige und geringe Förderbeträge gezahlt (Iwata 2003; Othake 2004). Hier ist ebenso wie in Großbritannien der Interventionsgrad eher gering zu bewerten.

\subsection{AKTIVE ARBEITSMARKTPOLITIK - KEINE KONVERGENZ DER SYSTEME}

Die mit der Alterung und teilweise auch Schrumpfung der Erwerbsbevölkerung einhergehenden Veränderungen auf dem

\footnotetext{
2 Im Rahmen der angeführten „Initiative 50plus" der Bundesregierung ist eine Modifizierung der Entgeltsicherung durch die Einführung eines übergreifenden Kombilohnmodells geplant.
} 
Arbeitsmarkt führen in den Untersuchungsländern zu einer Intensivierung der arbeitsmarktpolitischen Maßnahmen für ältere ArbeitnehmerInnen. Ebenso wie aber in der Vergangenheit der länderübergreifende säkulare Trend zur Frühverrentung von Spezifika des jeweiligen Wohlfahrtsstaates bestimmt war (vgl. hierzu jüngst noch Ebbinghaus 2006), ist auch diese arbeitsmarktpolitische Neuausrichtung von divergenten wohlfahrtsstaatlichen Strategien geprägt. Auch die in allen drei Ländern zu beobachtende Tendenz hin zu einer stärker „aktivierenden“ Ausrichtung der Arbeitsmarktpolitik kann nicht über die weiterhin bestehenden zentralen wohlfahrtsstaatlichen Unterschiede hinwegtäuschen.

Im britischen, marktliberalen Ansatz ist nach wie vor nur in sehr begrenztem Rahmen - was sowohl den finanziellen Umfang als auch die Breite der Maßnahmen angeht - eine aktive Arbeitsmarktpolitik für ältere ArbeitnehmerInnen anzutreffen. Es kommt zwar auf den ersten Blick zu Ausweitungen und Innovationen, die insbesondere mit der Progammatik des New Deal 50plus verbunden sind. Doch trotz der im New Deal 50plus zusammengefassten bzw. intensivierten Beratungs-, Vermittlungs-, und Qualifizierungsmaßnahmen sind die Fördermöglichkeiten für ältere ArbeitnehmerInnen im Bereich der beruflichen Qualifizierung, der Wiedereingliederungshilfen und der beruflichen Rehabilitation nur gering ausgebaut. Hierin spiegelt sich ungebrochen eine auf die Marktkräfte und die Eigenverantwortung der Arbeitsuchenden ausgerichtete Förderpolitik wider. Von einem Richtungswechsel - etwa hin zu einem stärker sozialdemokratisch-universalistisch geprägten System - kann daher trotz des höheren Aktivierungsgrades und der verstärkten Ausrichtung auf die Bekämpfung sozialer Ausgrenzungen nicht gesprochen werden.

In Deutschland ist zwar weiterhin ein breites Maßnahmenspektrum mit zum Teil hohen finanziellen Aufwendungen für Lohnsubventionen älterer Arbeitsloser und für die Entwicklung neuer Programmelemente, wie z. B. die betriebliche Weiterbildungsförderung für ältere Beschäftigte und die Entgeltsicherung, vorhanden. Allerdings bestehen zum einen - als Spätwirkung des Frühverrentungsgeschehens weiterhin strukturelle Ausgrenzungen älterer ArbeitnehmerInnen aus Fördermaßnahmen fort - so insbesondere in der För- derung der beruflichen Weiterbildung. Die aktive Arbeitsmarktpolitik für ältere ArbeitnehmerInnen ist so gesehen immer noch von der konservativ-korporatistischen Richtungsentscheidung zur Angebotsreduzierung geprägt. Zum anderen hat auch der Richtungswechsel hin zu einer „aktivierenden“ Arbeitsmarktpolitik tendenziell zu einer Ausgrenzung insbesondere von schwer vermittelbaren älteren Langzeitarbeitslosen geführt, und zwar vor allem in den Vermittlungsaktivitäten der Agenturen für Arbeit, den Trainings- und Qualifizierungsmaßnahmen und durch den Abbau von Arbeitsplatzbeschaffungsmaßnahmen.

In Japan wird weiterhin versucht, über die staatliche Förderung der Weiterbeschäftigung im Betrieb - bzw. der Subventionierung betrieblicher Altersgrenzen die Integration älterer ArbeitnehmerInnen in die Erwerbsarbeit sicherzustellen. Durch dieses auch als „staatsinterventionistisch“ zu kennzeichnende Vorgehen unterscheidet sich Japan stark von liberalen Wohlfahrtsstaaten, die nur sehr gering intervenieren. Diesem relativ hohen Aktivitätsgrad in der betrieblichen Beschäftigungssicherung der vorwiegend männlichen Kernbelegschaften im System der „lebenslangen Beschäftigung" stehen unterentwickelte Vermittlungs-, Qualifizierungsund Wiedereingliederungshilfen für ältere Arbeitslose gegenüber. In der aktiven Arbeitsmarktpolitik wird dem Problem der offenen Arbeitslosigkeit damit nur unzureichend begegnet. Dies belegt die Kontinuität der „hybriden“ Mischform der japanischen Arbeitsmarktpolitik für ältere ArbeitnehmerInnen, die Interventionsansätze seitens des Staates mit starken Marktelementen kombiniert.

\section{1}

\section{Ausblick - Neue Arbeits- marktrisiken für ältere ArbeitnehmerInnen}

Der demografische Wandel in der Erwerbsarbeit determiniert noch keineswegs einen arbeitsmarktpolitischen Automatismus hin zu einer verstärkten Förderung der Beschäftigungsfähigkeit und Arbeitsmarktintegration älterer ArbeitnehmerInnen. Im Gegenteil: Vor dem Hintergrund spezifischer Unterschiede der Wohlfahrtsstaatsregime reagiert die Arbeits- marktpolitik im Rahmen ihrer jeweiligen Systemlogik auf die Herausforderungen, und es entstehen spezifische neue Gefährdungen der Lebenslage älterer ArbeitnehmerInnen.

In Deutschland ist angesichts der Anhebung der Altersgrenzen und des Abbaus sozialer Unterstützungsleistungen bei Arbeitslosigkeit ein hoher existenzieller Druck auf die Erwerbstätigkeit im Alter ausgeübt worden. Sozialverträgliche Ausgliederungspfade für Problemguppen - insbesondere für ältere, gesundheitlich eingeschränkte Arbeitslose oder niedrig Qualifizierte existieren kaum noch. Ältere Beschäftigte, die aufgrund von betriebsbedingten Kündigungen, Betriebsaufgabe und ähnlichen Faktoren arbeitslos werden, müssen dem Arbeitsmarkt damit länger zur Verfügung stehen. Wenn sie aber weder qualifikatorisch noch gesundheitlich dazu in die Lage versetzt werden, besteht die Gefahr, dass die ohnehin schon hohe Dauer des Verbleibs in Arbeitslosigkeit weiter verlängert wird. Die derzeit an Konzepte der Aktivierung und zugleich an hohen Eingliederungsquoten orientierte Arbeitsmarktpolitik in Deutschland grenzt ältere Arbeitslose derzeit aber noch tendenziell aus Vermittlungs-, Trainings- und Qualifizierungsmaßnahmen aus.

In Japan ist von einer weiteren Zunahme irregulärer Beschäftigungsformen für ältere ArbeitnehmerInnen in Form von Teilzeitarbeit und befristeten Arbeitsverhältnissen mit niedrigem Lohn- und Absicherungsniveau auszugehen. Hierfür ist nicht nur die absehbare Fortführung der Praxis betrieblicher Altersgrenzen im $\mathrm{Zu}$ sammenwirken mit dem demografisch bedingten Angebotsdruck verantwortlich zu machen. Hinzu kommt vielmehr, dass auch staatlicherseits die Altersgrenzen für die bisher als Kompensation zur betrieblichen Ausgliederungspraxis gewährten Sonderaltersrenten seit dem Jahr 2001 sukzessive von 60 auf 65 Jahre angehoben werden.

In Großbritannien zeichnet sich eine Verschärfung der ohnehin hohen sozialen Ungleichheit unter den älteren ArbeitnehmerInnen ab. Hier sind bereits jetzt diejenigen, die von den Betrieben mit Hilfe der betrieblichen Altersvorsorge ausgegliedert werden, deutlich besser gestellt als die älteren Arbeitslosen, die auf die niedrige und stärker als in Deutschland bedarfsgeprüfte Arbeitslosenunterstützung angewiesen sind. Die geplante Erschwerung des Zugangs bei Erwerbsunfähigkeitsrenten dürf- 
te zukünftig bei wieder ansteigender Arbeitslosigkeit den Bestand an älteren Langzeitarbeitslosen deutlich erhöhen und diese Segmentierungen auf dem Arbeitsmarkt weiter verstärken.

Kurzschlüssige Argumentationsfiguren, die etwa aus dem hohen Beschäftigungsstand der älteren japanischen oder englischen ArbeitnehmerInnen auf erfolg- reiche arbeitsmarktpolitische Maßnahmetypen schließen und deren Übertragung propagieren, verkennen die weiter bestehenden Arbeitsmarktrisiken und sind eher kontraproduktiv. Die Systemdivergenzen schließen ein „policy-borrowing“ im Sinne der Übernahme einzelner arbeitsmarktpolitischer Programmpakete zwar nicht grundsätzlich aus. Dieses wäre allerdings sehr viel voraussetzungsvoller, als es bisher in der arbeitsmarktpolitischen Landschaft diskutiert wird. Und es setzt die Reflektion des jeweiligen wohlfahrtsstaatlichen Gesamtkontextes und der damit einhergehenden, je spezifischen Arbeitsmarktrisiken voraus.

\section{ITERATUR}

AEDSC (Association of Employment Development for Senior Citizens) (2002): Handbook of Labour Statistics on Aged Society, Tokyo Araki, T. (2002): Labor and Employment Law in Japan, Tokyo Atkinson, J./Evans, C./Willison, R./Lain, D./Van Gent, M. (2003): New Deal 50plus: Sustainability of employment, WAE142, London BA (Bundesanstalt für Arbeit) (2003): Strukturanalyse: Ältere Arbeitslose, Nürnberg

BA (Bundesanstalt für Arbeit) (2004): Eingliederungsbilanz 2003, Bundesergebnisse, Nürnberg

Barham, C. (2002): Labour market and demography project, in: Labour market trends 3, pp. 151-158

Bertelsmann Stiftung/BDA (Bundesvereinigung der Deutschen Arbeitgeberverbände) (Hrsg.) (2003): Beschäftigungschancen für ältere Arbeitnehmer - Internationaler Vergleich und Handlungsempfehlungen, Gütersloh

BMAS (Bundesministerium für Arbeit und Soziales) (2006): Initiative 50plus. Eckpunkte zur Erhöhung der Beschäftigungsfähigkeit und der Beschäftigungschancen älterer Menschen in Deutschland, Berlin Brussig, M./Knuth, M./Schweer, O. (2006): Arbeitsmarktpolitik für ältere Arbeitslose. Erfahrungen mit "Entgeltsicherung" und "Beitragsbonus", IAT-Report 02, Gelsenkirchen

Cabinet Office (2000): Winning the Generation Game - Improving Opportunities for People Aged 50-65 in Work and Community Activity, London

Casey, B. (2006): The employment of older people: can we learn from Japan?, in: Geneva Association Information Newsletter 38, pp. 3-7 Comptroller and Auditor General (2004): Welfare to Work: Tackling the Barriers to the Employment of Older People, HC 1026 Session 20032004, London

Deutscher Bundestag (2006): Bericht 2005 der Bundesregierung zur Wirksamkeit moderner Dienstleistungen am Arbeitsmarkt, Berlin DfEE (Department for Education and Employment) (2001): Training Older Workers, London DWP (Department for Work and Pensions) (2002a): Simplicity, security and choice: Working and saving for retirement, London DWP (Department for Work and Pensions) (2002b): Pathways to work: Helping people into employment. Cm 5690, London

DWP (Department for Work and Pensions) (2003): Pathways to work: Helping people into employment. The Government's response and action plan. Cm 5830, London.

DWP (Department for Work and Pensions) (2006): A new Deal for welfare: Empowering people to work, London

Ebbinghaus, B. (2006): The reform of early retirement in Europe, Japan and the USA, Oxford
Esping-Andersen, G. (1990): The Three Worlds of Welfare Capitalism, Princeton

Esping-Andersen, G. (1997): Hybrid or Unique? The Distinctiveness of the Japanes Welfare State, in: Journal of European Social Policy 7/3, pp. 179-189

Esping-Andersen, G. (1999): Social Foundations of Postindustrial Economies, Oxford

ETUI (European Trade Union Institute) (ed.) (2002): Active Strategies for Older Workers, Brussels

Expertenkommission (Expertenkommission Finanzierung Lebenslanges Lernen) (2004): Finanzierung Lebenslangen Lernens - der Weg in die Zukunft, Schlussbericht, Bielefeld

Frerichs, F./Taylor, P. (2005): Labour market policies for older workers and demographic change. A comparative analysis of policy approaches in Germany and the United Kingdom, London

Gemeinsame Erklärung (2001): Gemeinsame Erklärung des Bündnisses für Arbeit, Ausbildung und Wettbewerbsfähigkeit zu den Ergebnissen des 7. Spitzengespräches am 4. März 2001, Berlin

Higuchi, Y. (2004): Employment Strategies Required in an Ageing Society with Fewer Children, in: Japan Labour review 1, pp. 17-8

Hirsch, D. (2003): Crossroads after 50. Improving choices in work and retirement, Bristol

Hoffmann, E./Walwei, U. (2002): Wandel der Erwerbsformen - Beschäftigungssituation von Frauen in Deutschland, in: Engelbrech, G. (Hrsg.): Arbeitsmarktchancen für Frauen, BeitrAB 258, Nürnberg, S. 67-92 Iwata, K. (2002): Policies for the Employment of Older Persons in Japan. A Comparison between the EU, US and Japan. Journal of Japanese Trade \& Industry, 3, pp. 12-16

Iwata, K. (2003): Labor Market Policies in the Era of Population Aging: Japan's Case, Tokyo

Maltby, T./de Vroom, B./Mirabile, M. L./Overbye, E. (eds.) (2004):

Ageing and Transition to Retirement. A Comparative Analysis of European Welfare States, Aldershot

Ministry of Labour (2000): White Paper on Labour 2000. The Best Mix of Young and Middle-Aged and Older Workers in an Aging Society, Tokyo MHLW (Ministry of Health, Labour and Welfare) (2005): White Paper on the Labour Economy, Tokyo

Moss, N./Arrowsmith, J. (2003): A Review of "What Works" for Clients Aged Over 50, London

Naganawa, H. (2002): Re-employment of older white-collar workers, in: Japan Labor Bulletin 2, pp. 6-10

OECD (2001): Ageing and Income. Financial Resources and Retirement in 9 OECD Countries, Paris 
OECD (2003): Economic Outlook, Paris

OECD (2004a): Ageing and Employment Policies, United Kingdom, Paris

OECD (2004b): Ageing and Employment Policies, Finland, Paris.

OECD (2004c): Ageing and Employment Policies, Japan, Paris

OECD (2005): Alterung und Beschäftigungspolitik, Deutschland, Paris

OECD (2006a): Live longer, work longer, Paris

OECD (2006b): Economic Survey of Germany 2006, Paris

Ohtake, F. (2004): Structural Unemployment Measures in Japan, in:

Japan Labor Review 2, pp. 28-53

Pascha, W. (2003): Wirtschaft, in: Kevenhörster, P./Pascha, W./Shire,

K. A. (Hrsg.): Japan: Wirtschaft - Gesellschaft - Politik, Opladen,

S. 13-179

Prager, J. U./Schleiter, A. (Hrsg.) (2006): Länger leben, arbeiten und sich engagieren. Chancen werteschaffender Beschäftigung bis ins Alter, Gütersloh

Schütz, H./Oschmiansky, F. (2006): Arbeitsamt war gestern. Neuausrichtung der Vermittlungsprozesse in der Bundesagentur für Arbeit nach den Hartz-Gesetzen, in: Zeitschrift für Sozialreform 1, S. 5-28
Seike, A. (2001): Beyond Lifetime Employment, in: Geneva Papers on Risk and Insurance 4, pp. 642-655

Shire, K. A. (2003): Gesellschaft, in: Kevenhörster, P./Pascha, W./Shire, K. A. (Hrsg.): Japan: Wirtschaft - Gesellschaft - Politik, Opladen, S. 179258

Sproß, C./Eichhorst, W. (2005): Beschäftigung Älterer. Bislang noch kein Paradigmenwechsel, in: Bundesarbeitsblatt 11, S. 4-12

Statistisches Bundesamt (2003): Bevölkerung Deutschlands bis 2050, 10. Koordinierte Bevölkerungsvorausberechnung, Wiesbaden Taylor, P. (2002): New policies for older workers, Bristol Taylor, P. (2006): Employment initiatives for an ageing workforce in the EU 15, Dublin

Waldenberger, F. (2003): Japans Arbeitsmarktlage und Arbeitsmarktpolitik aus deutscher Sicht, in: Conrad, H./Kroker, R. (Hrsg.): Deutschland und Japan. Mit Reformen zu neuer Dynamik, Köln, S. 103-128 Walker, A. (2002): Active strategies for older workers in the UK, in: Epsen, M./Foden, D./Hutsebaut, M. (eds.): Active strategies for older workers, Brussels, pp. $403-435$

\section{Über "Hartz" hinaus - Stimmt die Richtung in der Arbeitsmarktpolitik?}

Fachtagung des Projektverbunds Monitor Arbeitsmarktpolitik

\section{9./30. März 2006 in Berlin}

Eine Zwischenbilanz fünf Jahre nach „Hartz“ sowie die von der Politik auf die Tagesordnung gesetzte Neugestaltung der Arbeitsmarktpolitik setzen ein Gespräch darüber voraus, welche Risiken Arbeitsmarktpolitik unter veränderten ökonomischen und sozialen Bedingungen bearbeiten kann und soll. Die Debatte über vorliegende Befunde der Wirkungsforschung ist dafür eine Grundlage. Im Mittelpunkt der Tagung steht jedoch nicht die Vermittlung der Evaluationsergebnisse. Das Anliegen ist vielmehr, über eine kritische Auseinandersetzung mit den Evaluationsbefunden zu einer Diskussion über Ziele, Funktionen und Aufgaben der Arbeitsmarktpolitik und über die Anforderungen an die Wirkungsforschung zu kommen.

Programm

I. Wandel der Funktion von Arbeitsmarktpolitik

Bernd Reissert (Gründungsrektor der Hochschule der Bundesagentur für Arbeit),

Jürgen Kühl (ehemals IAB und Wirtschafts- und Arbeitsministerium Thüringen), Alexandra Wagner (FIA)

II. Arbeitsmarktpolitik und Existenzsicherung

Werner Eichhorst (IZA), Irene Becker (Uni Frankfurt/M.), Judith Aust (WSI)

III. Arbeitsmarkpolitik in zwei Regelkreisen

Hugh Mosley (WZB), Johannes Jakob (DGB), Till Müller-Schoell (WSI)

IV. Mehr als FbW - Qualifizierung in der Arbeitsmarktpolitik

Edgar Sauter (Anerkennungsbeirat der BA, früher Bibb), Siegfried Schmauder (Bundesverband der Träger

beruflicher Bildung), Volker Baethge-Kinsky (SOFI)

V. Reflexion - Zukunft der Arbeitsmarktpolitik

Rolf Schmachtenberg (BMAS, angefragt), Matthias Knuth (IAQ, angefragt), Anne Ames (BAG der Erwerbsloseninitiativen), Moderation: Erika Mezger (HBS)

Weitere Informationen im Internet unter: www.monapoli.de

Anmeldung: Christian Baum, Hans-Böckler-Stiftung, Hans-Böckler-Str. 39, 40476 Düsseldorf, 0211/7778-123 